\begin{tabular}{|c|l|}
\hline Title & Movement of Small A ngle Boundary of Ice Crystal \\
\hline Author(s) & Higashi, A kira; Sakai, Naomi \\
\hline Citation & 北海道大學理學部紀要, 5(5), 221-237 \\
\hline Issue Date & 1961 \\
\hline Doc URL & http://hdl.handle.net/2115/34238 \\
\hline Type & bulletin (article) \\
\hline File Information & 5_P221-237.pdf \\
\hline
\end{tabular}

Instructions for use 


\title{
Movement of Small Angle Boundary of Ice Crystal
}

\author{
Akira HIGASHI and Naomi SAKaI
}

(Received Nov. 1, 196k)

\begin{abstract}
The stress induced movement of small angle boundaries in ice crystals was investigated with specimens of natural single crystals. Boundaries made by bending bars of ice were observed under microscope through crossed polaroids. Dependence of the rate of movement upon temperature, applied stress and angle of misfit was obtained. The activation energy for the rate of movement takes a value of about $12 \mathrm{Kcal} / \mathrm{mol}$ to $17 \mathrm{Kcal} / \mathrm{mol}$ with changing angles between the direction of length and that of the crystal a-axis. This variation was semiquantatively interpreted by a simple dislocation model of the boundary in the ice crystal.
\end{abstract}

\section{Introduction}

Though the movement of small angle boundary is believed to be a very powerful tool for study of the dislocation mechanism of slip in crystal, ${ }^{1}$. it has not been investigated very widely except in the case of zinc. As a first part of our extensive studies of slip mechanism in ice crystal, the stress induced movement of small angle boundary was investigated with specimens of natural single crystals.

As is the case of zinc, the ice crystal has a basal slip plane that makes this kind of study easy. Due to its transparancy, ice crystals have more advantages for observation of the movement of boundary than do zinc crystals. Simple methods of determining the a-axis of ice crystals by Tyndall figure etc. make it easy to form the boundary which has an arbitrary angle with the a-axis in the basal plane of ice crystal.

In the present study of the movement of small angle boundary, it was aimed to make clear the nature of dislocations which should exist in ice crystals and move to the boundary when it is made. Experiments performed for this purpose include dependences of the rate of movement upon temperature, applied stress and angle of misfit. Effect of reversal of the direction of movement in a crystal was also examined. Utilizing an advantage stated above, study was made of the variation of the activation energy for the rate of movement with the change of horizontal directional angle between the crystal a-axis and the direction of length of specimen. This can give some useful items of informatin about any mechanism of dislocation motion in the ice crystal. However, results of experiments are not necessarily consistent for building up 
a simple mechanism of dislocation motion. Further theoretical considerations should wait until more informations of slip in the ice crystal is obtained by means of various kinds of experiments.

\section{Experimental Procedure}

\section{a) Material and preparation of ice specimens}

The small angle boundaries for study were made in a rectangular bar of single crystal of ice. The specimens of $1 \times 1 \mathrm{~cm}^{2}$ in cross section and $10 \mathrm{~cm}$ in length were cut from large natural single crystals. These crystals were brought from the Mendenhall Glacier, Alaska in 1960 by the efforts of the Alaskan Glacier Expedition of Hokkaido University headed by the senior author. The qualities of crystals are believed to be the best in the world in either natural or artificially made single crystals of ice. Electrical conductivity of melt water of the crystal is the order of $1 \mu \Omega / \mathrm{cm}^{2}$ which fact reveals that the impurity content is very small. Density of the crystal measured previously by BuTkovitch was $0.917 .{ }^{3)}$ This shows that the crystal has neither air void nor large imperfections which should appear as deficit of the density. Optical examination using polaroids revealed no substructure in the crystal at all.

Rectangular bars were cut from the single crystals by a goniometric bandsaw. Crystallographic axes were determined by the methods of Tyndall figure, ${ }^{4)}$ thermal etch $\mathrm{pit}^{5)}$ and also by the reflection from surface frosts which had grown over the crystal. ${ }^{6}$. Error of determination of the axes is within about $1^{\circ}$ when the last method was adopted. Bars cut with the saw a little larger than the proposed size were scraped with a plane until the final desired cross section was obtained.

\section{b) Method of making a small angle boundary}

The small angle boundary was made at the center of each specimen of a rectangular bar by the method of bending. The bar has its long axis perpendicular to the crystal c-axis and its top and bottom surfaces are parallel to the base plane. Direction of the long axis was varied in respect of the a-axis of the crystal. As the ice crystal has a basal slip plane, the bending causes glide in the crystal most easily in this type of specimen.

Bending was carried out by central loading of a specimen supported by two wedges of about $52 \mathrm{~mm}$ distance apart. Temperature at which the bending was carried out was about $-10^{\circ} \mathrm{C}$ with fluctuations of a few degrees due to the temperature variation of the cold room. Angle of bending or the angle of misfit at the boundary was controlled by the duration of bending and also by the weight. For example, an angle of $2^{\circ}$ was obtained in about 20 


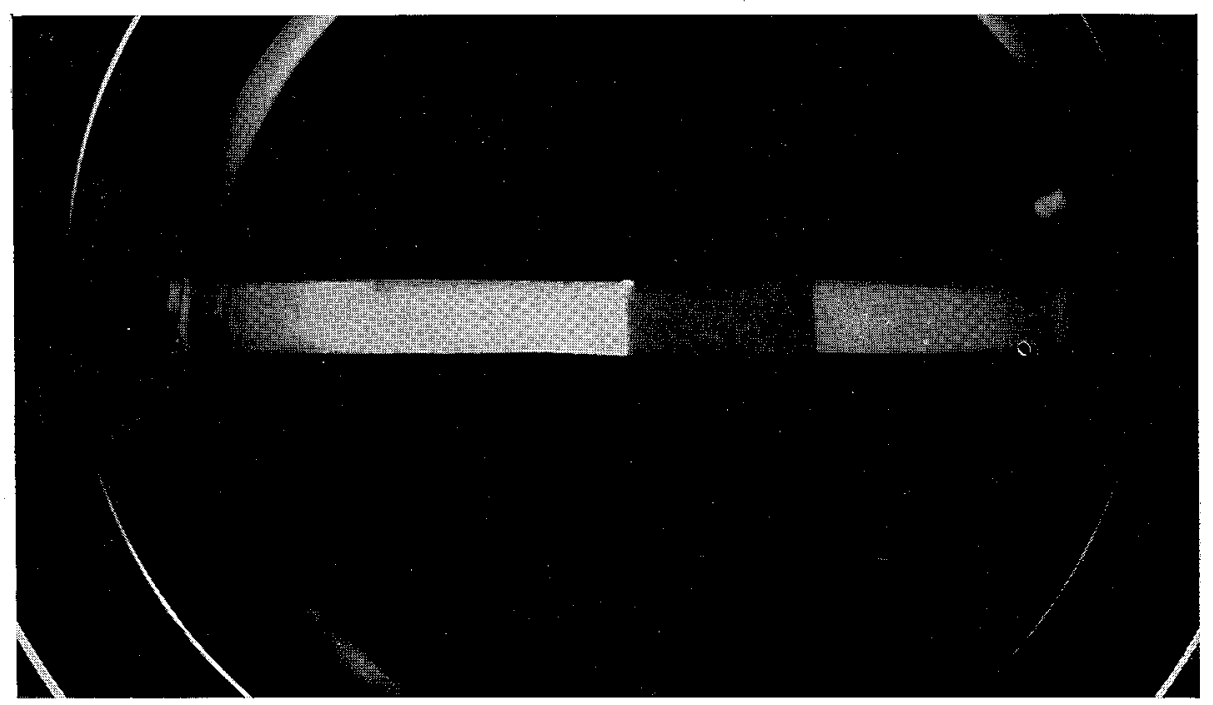

Fig. 1. Ice Specimenin in which small angle boundaries were produced by bending. Photograph taken through crossed polaroids.

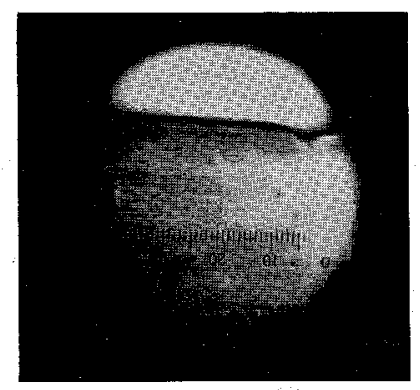

1) $\mathrm{t}=20 \mathrm{~min}$

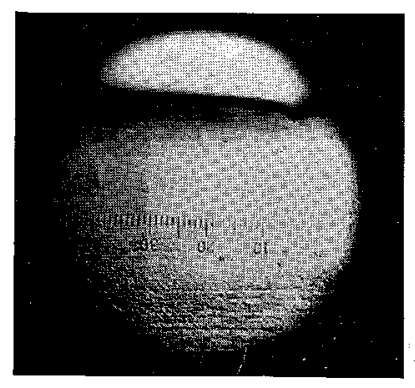

4) $t=90 \mathrm{~min}$.

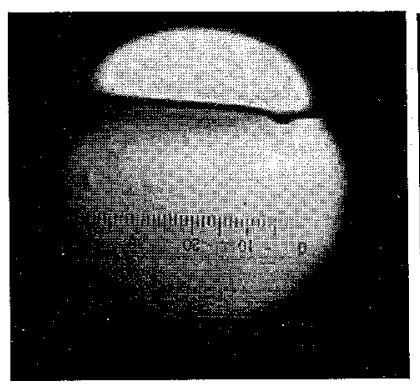

2) $t=40 \mathrm{~min}$.

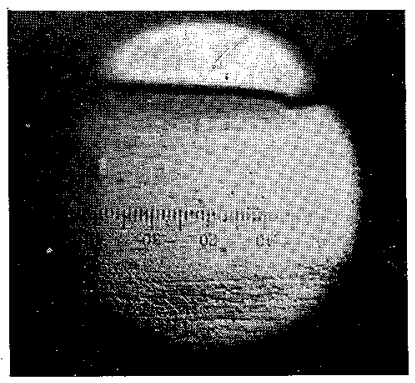

5) $t=110 \mathrm{~min}$.

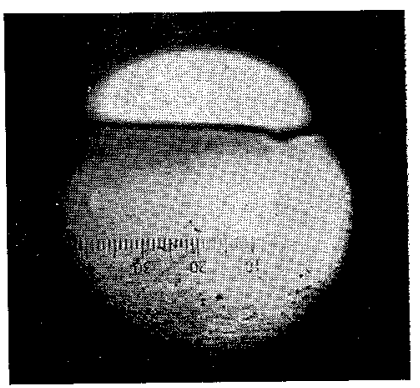

3) $t=70 \mathrm{~min}$.

$\mathrm{t}$ : time ellapsed

Weight $300 \mathrm{~g}$

Temp. $\quad-5.5^{\circ} \mathrm{C}$

Fig. 3. Process of the movement of a small angle boundary. Photomicrographs through a microscope with a graded eyepiece. One division of the scale is $1 / 16 \mathrm{~mm}$. 
hours with $2 \mathrm{~kg}$ of weight.

The boundaries thus made at the center and also at the position of supporting wedges in the bar are clearly visible due to different transparency of both sides of each boundary when the bar was examined through crossed polaroids from the side. An example of the bent specimen is shown in the photograph of Fig. 1. Contrast of the transparancy increases with the increase of angle of bending thus rendering easy the observation of the boundary. When the bending angle is less than $1^{\circ}$, the contrast becomes too weak to identify enable clear identification of the boundary On the other hand, when the bending angle is increased over about $10^{\circ}$, the boundary splits in two or three lines which divide that portion into small trianglular areas of different transparancy. In this case the boundary is no more a small angle boundary.

\section{c) Observation of the movement of boundary}

The boundary produced at the center of bar as stated in b) was used for experiments on the movement. The bar was clamped at one end horizontally and was pulled down by weights at other end making a cantilever bending. Edge of the clamp and the position of hanging weights at opposite end were both about $23 \mathrm{~mm}$ from the center of the bar. This means that the boundaries

(A)

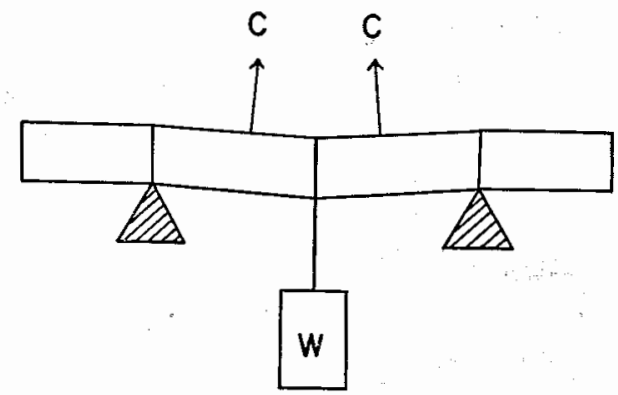

(B)

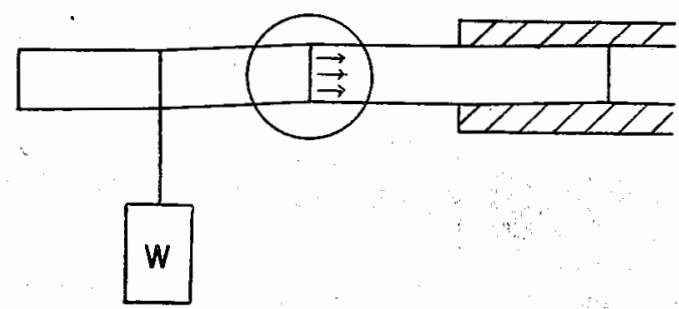

Fig. 2. Methods of producing small angle boundaries by bending (A) and disposition of a specimen for the experiment of movement (B). 
at the position of wedges at both ends were completely out of consideration in the experiment. At this disposition, the top and bottom sides of the bar in preparation of the specimen were reversed as shown schematically in Fig. 2. The clamped bar was put between crossed polaroids at the sides.

Stress induced movement of the small angle boundary because of the cantilever bending was measured by observing the line of contrast through a horizontal microscope with a scale in the eyepiece. An example of the movement of boundary is illustrated in photographs of Fig. 3. A temperature-box put in a cold room laboratory was used to keep constant the temperature of the specimen together with that of the microscope and polaroids. Deviation of the temperature from the mean value was about $\pm 0.1^{\circ} \mathrm{C}$.

\section{Results of the experiments}

\section{a) Time-displacement curves}

Several examples of the time-displacement curve of the small angle boundary are given in Fig. 4. As can be seen in this figure, movement of the boundary is slow at first for at laest about 20 minutes. This probably means that there exists an incubation time during which the rate of movement increases gradually

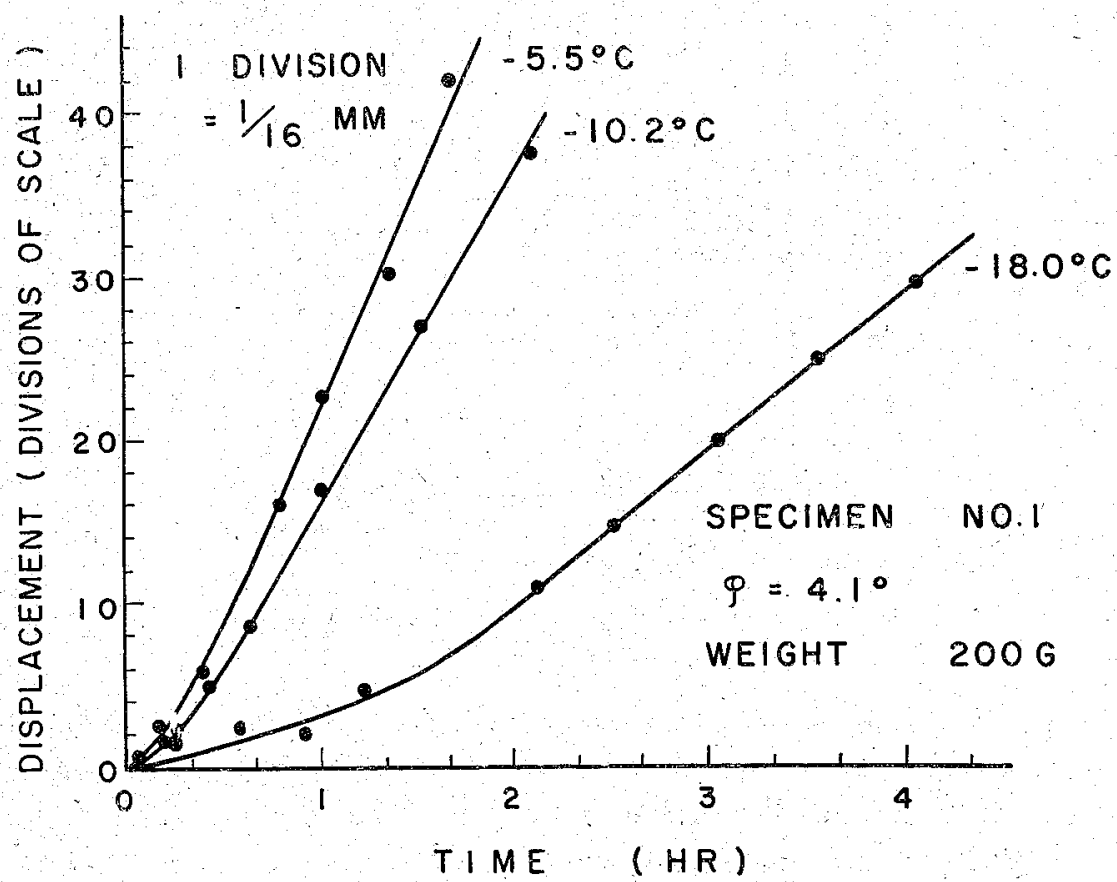

Fig. 4. Examples of time-displacement curves. 
to its constant rate. Though it has been considered later that the incubation time may have some significant connections with the dislocation mechanism of the movement of a small angle boundary, this henomenon was disregarded in the present study. Consequently, only the steady rate of movements of the boundary after this period was taken into account. The rate of movement was calculated as a gradient of each straight line of the time-displacement diagram shown in Fig. 4.

\section{b) Relationship between the rate of movement and the angle of bending}

Dependency of the rate of movement upon the angle of bending was examined with specimens of different angles of bending at two different temperature. Results of the expeniments are shown in Fig. 5. It is concluded that the rate of movement decreases almost linearly as the angle of bending increases.

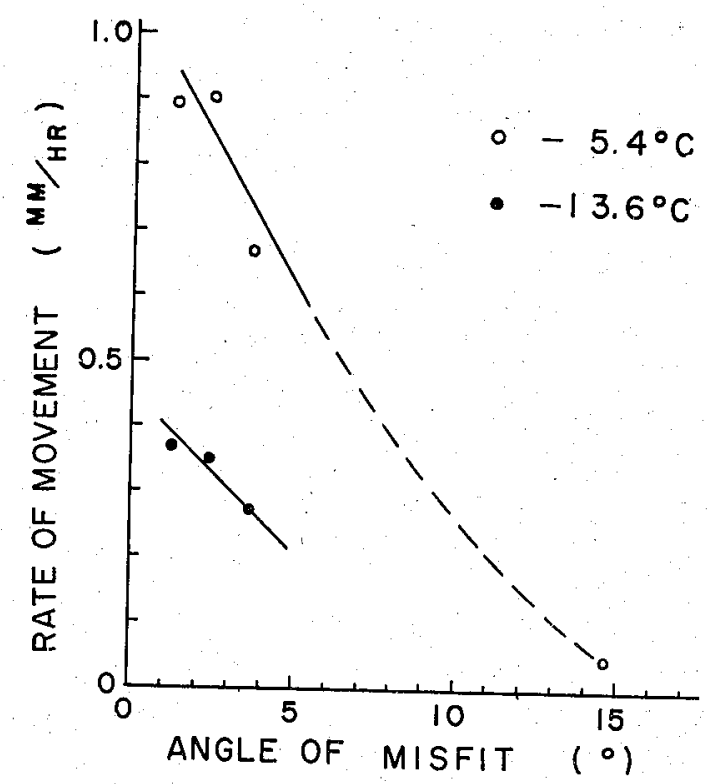

Fig. 5. Relationship between the rate of movement. and the angle of misfit.

As stated before, the boundary splits into two or three lines and each of them moves differently, when the angle exceeds about $10^{\circ}$. Therefore, a dot near $15^{\circ}$ in the figure has little value in determining the relation. Polygonization was observed near the boundary when the angle of bending was as large. as exceeding $10^{\circ}$. This was revealed by rows of each pits along the boundary 


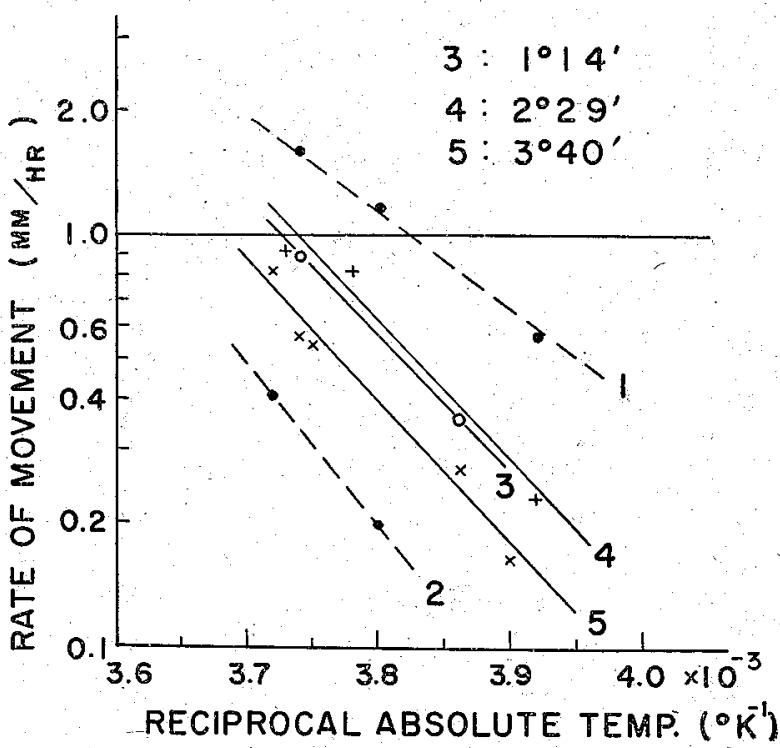

Fig. 6. Temperature dependence of the rate of movement with specimens not controlled about the directional angle $\theta$.

which were observable through an optical microscope. But details of the observation will be presented in a separate paper.

\section{c) Temperature dependence of the rate of movement}

Temperature dependence of the rate of movement was measured in a range between about $-5^{\circ} \mathrm{C}$ and $-25^{\circ} \mathrm{C}$. At a lower temperature than this range, the rate of movement was so slow that it was difficult to measure the rate accurately. Measurements were carried out at three or four temperatures for each specimen.

Results of experiments are illustrated in Fig. 6 and Fig. 7 in both of which the rate of moving versus the reciprocal absolute temperature is graphed. Fig. 6 is for the first five specimens No. 1 to No. 5. Of these specimens, the angle of direction of long axis in respect of a-axis was not known except that the three specimens No's. 3,4 and 5 were cut parallel from one block of crystal. These three were used for the comparison of different angles of bending. Parallel straight lines for these three specimens indicate that the activation energy for the rate of movement does not differ with the difference of angle of bending. The straight line of No. 2 is almost parallel to the three stated above. Activation energy for these four specimens is calculated as about 17 $\mathrm{Kcal} / \mathrm{mol}$. On the other hand, the straight line for No. 1 is very different from those of the above four and the activation energy calculated from it is about $12 \mathrm{Kcal} / \mathrm{mol}$. From such difference in the activation energy, the authors has a 


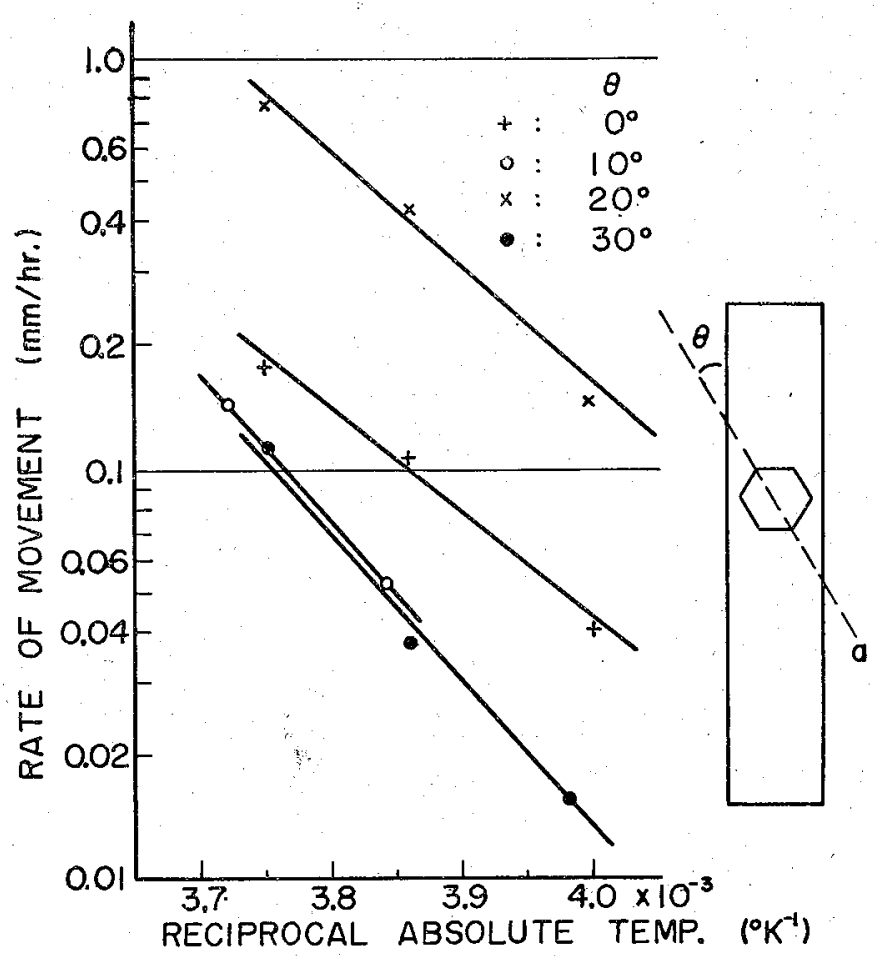

Fig. 7. Temperature dependence of the rate of movement with specimens of various directional angle $\theta$.

suspicion that the activation energy might depend on the direction of the long axis of specimens in respect to a-axis of the crystal.

Shown in Fig. 7 is a diagram of temperature versus the rate of movement from which the dependence of the activation energy upon the direction of long axis of specimens was derived. This is the results of measurements on four specimens of different angles $\theta$ between the crystal a-axis and the long axis of the specimen. These specimens were cut from one block of a single crystal. The angle $\theta$ is deliberately changed from $0^{\circ}$ to $30^{\circ}$ with $10^{\circ}$ intervals.

TABLE I. Variation of the activation energy $W$ for movement of small angle boundary with the change of directional angle $\theta$ between the crystal a-axis and the long axis of a specimen

\begin{tabular}{c|c|ccc}
\hline 0, degree & 0 & 10 & 20 & 30 \\
\hline$\therefore W, \mathrm{Kcal} / \mathrm{mol}$ & 11.6 & 16.6 & 12.9 & 16.6 \\
\hline
\end{tabular}




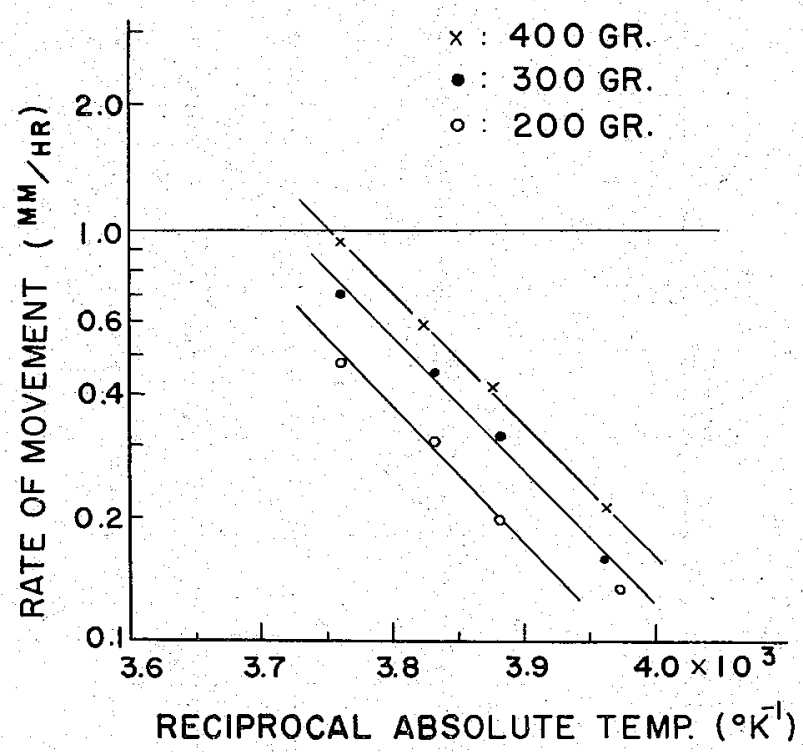

Fig. 8. Relationship between the rate of movement and the applied load.

Due to the symmetry of crystal axis, greater variation of the angle than $30^{\circ}$ is just a repetition of the same thing as between $0^{\circ}$ and $30^{\circ}$. Direction of the long axis when $\theta=30^{\circ}$ is that of $b$-axis of the crystal. Values of the activation energy calculated from the straight lines drawn in the figure are tabulated in Table $I$ in respect to the angle $\theta$. It is concluded on the basis of data in this table that the activation energy takes a value of about $12 \mathrm{Kcal} / \mathrm{mol}$ and 17 $\mathrm{Kcal} / \mathrm{mol}$ alternately with every $10^{\circ}$ change of the angle $\theta$.

\section{d) Stress dependnece of the rate of movement}

Stress dependence of the rate of movement was examined by experiments of two types: one by variying the weight of pull at the end of the ice specimien in cantilever position and other by varying the thickness of the specimen for each run of the measurements the weight being kept constant. In the first experiments specimens cut parallel to those of No. $3,4,5$ were used. Rate of movement versus reciprocal temperature relations for those experiments are illustrated in Fig. 8. Three straight lines correspond for $200 \mathrm{~g}, 300 \mathrm{~g}$ and $400 \mathrm{~g}$ from the bottom. As the slope of the line is the same for the three lines, the activation energy for the rate of movement does not vary with henchange of weight. Numerical value of the activation energy is $14.7 \mathrm{Kcal} /$ Nere the relation between the weight and the rate of 


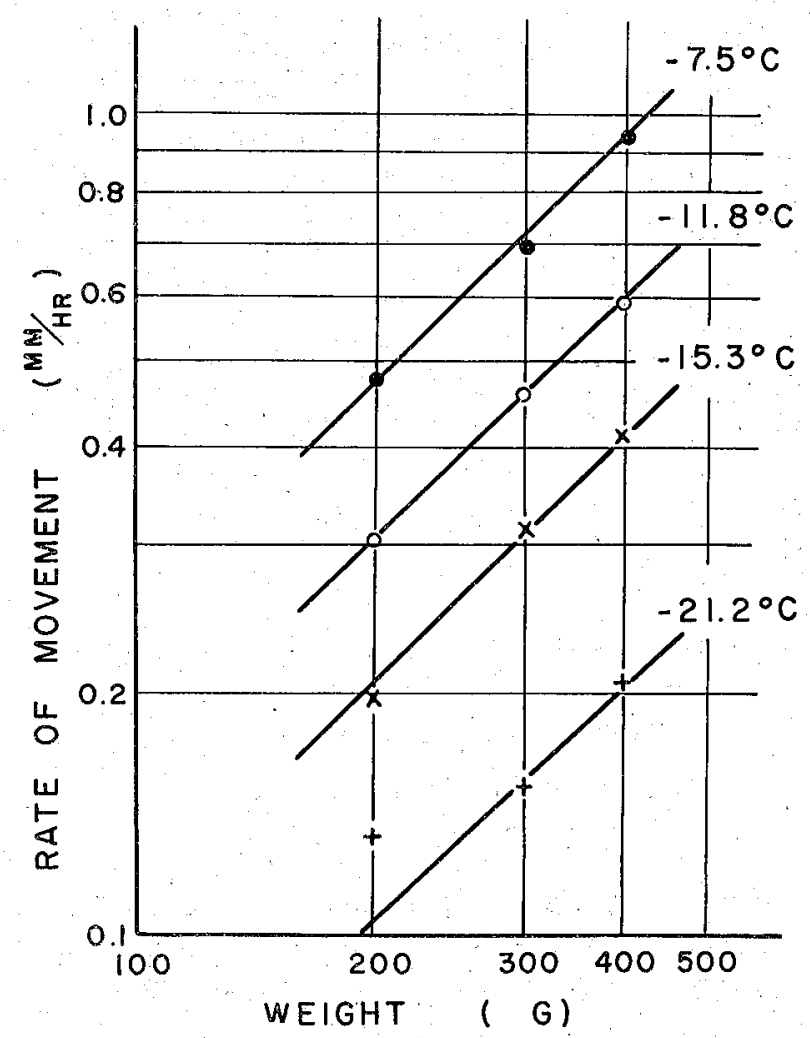

Fig. 9. Logarithmic plot of the load dependence at elifferent temperature.

movement is plotted on logarithmic graph paper, using the same data, the graph appears like that in Fig. 9. Disregarding one point of $200 \mathrm{~g}$ at $-21.2^{\circ} \mathrm{C}$, each line at about the same temperature has a slope of 45 degrees. This means that the rate of movement is proportional to the value of the weight.

The second type experiments of changing the thickness of specimens were carried out with two specimens having different angles of bending but at about the same temperature $-7.0^{\circ} \mathrm{C}$. The first specimen was used for three experiments at $11.4 \mathrm{~mm}, 9.0 \mathrm{~mm}$ and $7.2 \mathrm{~mm}$ thickness; it being made thinner by means of a plane between successive two experiments. The other specimen was furst used as $15.6 \mathrm{~mm}$ thickness with measurements at $13.4 \mathrm{~mm}, 11.4 \mathrm{~mm}$, $9.3 \mathrm{~mm}$ and $7.5 \mathrm{~mm}$ following in succession. Results of experiments are shown in Fig. 10 by means of logarithmic plots. The general tendency is that the rate of movement decreases with increase in thickness. By calculation of the slop of the curves in the figure, the following empirical formulae are obtained. 


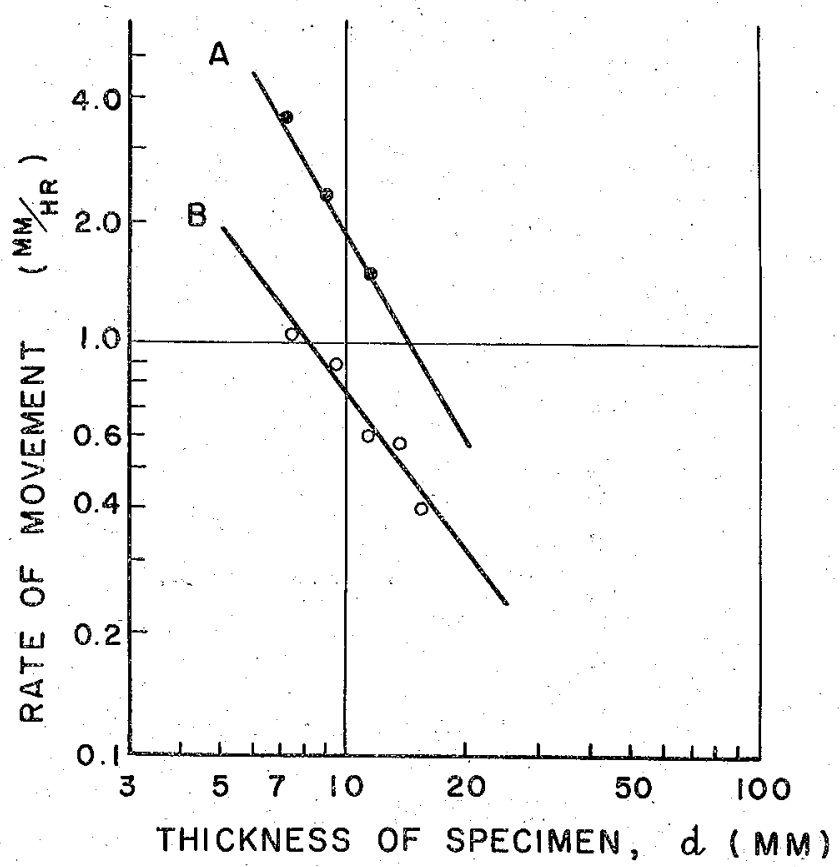

Fig. 10. Logarithmic plot of the thickness dependence.

$$
\begin{aligned}
& v=115(d)^{-18} \quad \therefore \quad \text { for A curve } \\
& v=15.5(d)^{-1.47} \quad \therefore \text { for B curve }
\end{aligned}
$$

Here, $v$ is the rate of movement expressed by $\mathrm{mm} / \mathrm{hr}$ and $d$ is the thickness in mm. The meaning of the numerals of the power in those formulae will be discussed later.

\section{e) Reversal of the direction of movement of the boundary}

Effect of reversal of the direction of motion was examined with a specimen of regular size with a load of $300 \mathrm{~g}$. The specimen with which the measurements of boundary motion to one direction (denoted + ) had been made was remounted on the clamp with reversal of the direction of its long axis. This reversed the direction of boundary motion (denoted-) in the crystal in the next experiments. This procedure was repeated four times. The rate of movemnt at each run of reversals is plotted against the number of the reversal in Fig. 11. This shows that the rate of movement decreases appreciably in - direction and then increases to a value near to that of the first time when the direction is reversed to + again. Accompanying this wàvy change, rate of movement decreases gradually on the whole. This gradual decrease is also a result of 


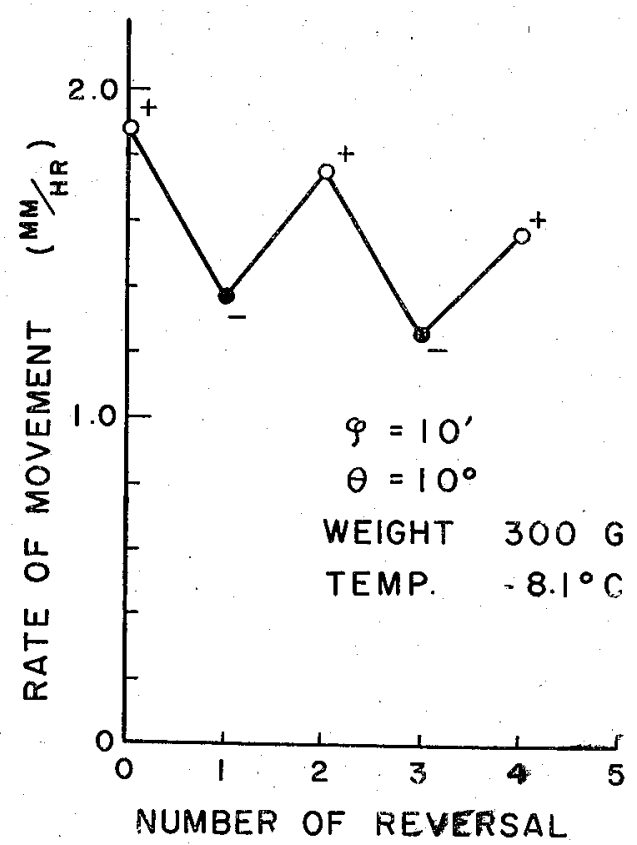

Fig. 11. Effect of the reversal firection of motion on the rate of movement of a small angle boundary.

reversal of the direction of metion, because the rate of movement did not change when the movement had been several times repeated in one direction without changing other conditions.

\section{a) General}

\section{Discussion}

In this investigation of the movement of small angle boundaries, splitting or union of boundary lines which could be seen in the region of a large angle of misfit were not considered as subjects of primary interest. But, these facts observed and also the fact that the rate of movement decreased with increasing misfit angle suggest that the boundaries are formed of arrays of edge dislocations of the same sign and that they are forming substructures in the crystal near a largle angle boundary.

The following discussion is restricted to the range of misfit angle within $2 \sim 3^{\circ}$. Even in this range, the rate of movement is rather slow though the temperature is as high as near the melting point.

\section{b) Activation energy of the rate of movement}

The activation energy of the movement takes a value of about $12 \mathrm{Kcal} / \mathrm{mol}$ 
and $17 \mathrm{Kcal} / \mathrm{mol}$ alternately with every $10^{\circ}$ change in the angle $\theta$ between the crystal a-axis and the long axis of a specimen (Table 1). As there are few other estimates of the activation energy of the process related to the basal slip in the single crystal of ice, it is difficult to derive a definite mechanism of dislocation motion at the moving boundary. But, it would be worth noting that the values of activation energy for dielectric relaxation ${ }^{8)}$ and for creep under low stress ${ }^{9}$ measured hitherto with polycrystalline ice lie within the range obtained in the present experiments.

If a simple dislocation model of the small angle boundary is adopted, dislocations at a plane of small angle boundary must move on the basal slip plane encountering barriers. So, activation energy of the rate of movement is considered as the activation energy for the thermal generation of kinks of dislocation under a stress. Variation of the activation energy with the change of $\theta$ could be interpreted by the direction dependence of that energy. For a simplicity of interpretation, the discussion will be limited in the difference between $11.6 \mathrm{Kcal} / \mathrm{mol}$ and $16.6 \mathrm{Kcal} / \mathrm{mol}$ which correspond to $\theta=0^{\circ}$ and $30^{\circ}$ respectively. As it is difficult to estimate the theoretical value of activating energy itself for each case, comparison will be done only with the ratio of the values for both cases above.

An equation for giving the activation energy $W$ for the dislocation motion based on the generation of kinks is

$$
W=a \sqrt{\frac{a b \sigma_{0} T}{2}}\left(1-y+\frac{1}{2} y \log y\right)
$$

where $a$ is the distance between the neighbouring valleys of dislocation lines, $b$ Burgers vector, $T$ line tension of a dislocation, $\sigma$ applied shear stress, $\sigma_{0}$ critical shear stress and $y=\sigma / \sigma_{0}$. Potential barrier assumed for deriving this equation has a type of

$$
V=\alpha(x-n a)^{2} \quad \text { for }|x-n a| \geq \frac{a}{2}
$$

Another equation of activation energy is

$$
W=\frac{4}{3} a \sqrt{a b \sigma_{0} T} \frac{\sqrt{1-y}}{1+y}
$$

for a potential barrier of a type of

$$
V=\alpha|x-n a| \quad \text { for } \quad|x-n a| \geq \frac{a}{2}
$$

These equations are modifications of those offered for crystals of diamond struc- 


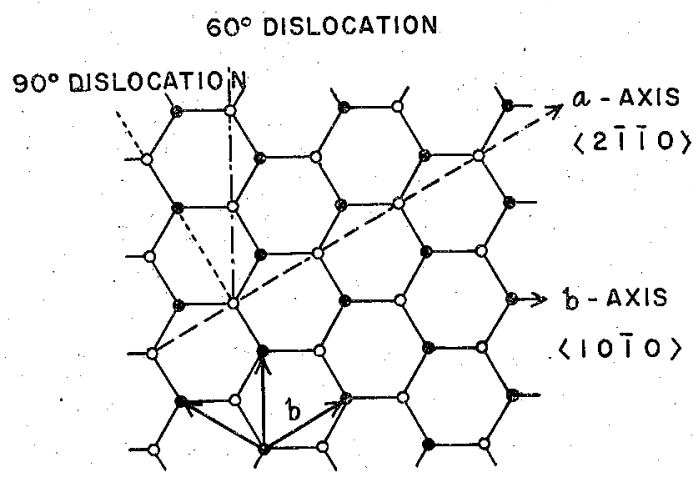

(A)

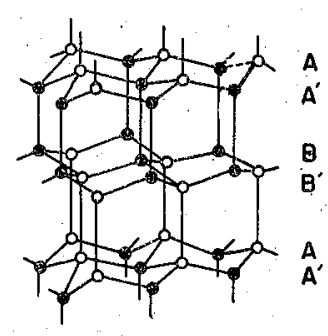

(B)

Fig. 12. Structure of ice crystal, basal plane (A) and side view (B).

ture by $\mathrm{H}$. SuzUKI. ${ }^{10}$ ) In either case, the activation energy is proportional to $a^{\frac{3}{2}}$, provided that the force to move dislocation $b \sigma_{0}$ and the line tension of a dislocation $T$ do not depend on the direction.

Fig. 12 (A) shows the atomic configuration of an ice crystal in its basal plane, indicating only oxygen atoms by circles and hydrogen bonds among them by solid lines. Open circles represent atoms at $\mathrm{A}$ or $\mathrm{B}^{\prime}$ planes and solid circles at $\mathrm{A}^{\prime}$ or $\mathrm{B}$ planes of the vertical rows of atoms as shown in Fig. 12 (B). Crystallographic a-axis is $\langle 2 \overline{1} \overline{1} 0\rangle$ direction, which is the connecting line of neighbouring two solid circles or two open circles. ${ }^{13}$ Apparently this is also the direction of Burgers vector on the basal slip plane. As the dislocation lines on the small angle boundary must lie perpendicular to the direction of movement, they should have direction of $\langle 10 \overline{1} 0\rangle$ when the direction of length of specimen coincides with the crystal a-axis. This is a pure edge dislocation or $90^{\circ}$ dislocation if the name is taken after Van Bueren. In the case of $\theta=30^{\circ}$, the dislocation line has a direction of $\langle 2110\rangle$, which makes a $60^{\circ}$ dislocation.

By a simple geometrical procedure, the distance $a$ in the case of $60^{\circ}$ dislocation is $\sqrt{3}$ times the distance $a$ in the case of $90^{\circ}$ dislocation. This means that the activation energy for the former should be $(\sqrt{3})^{\frac{2}{2}}=2.28$ times as large as the latter. Experimental result of this ratio calculated from Table 1 is $16.6 / 11.6=1.43$. Discrepancy between the theoretical and experimental values is not too large and it seems that the dependency of activation energy on the direction of the long axis of an ice specimen could be interpreted by a simple model of movement with the generation of kinks of edge dislocations on the 
basal slip plane.

Of course, the interpretation above is true for the first approximation. There are some other factors that should be considered; for example, such assumptions as that $b \sigma_{0}$ and $T$ do not depend on the direction are presumably too simple for a real mechanism of dislocation motion. It should be noted that there is a possibility that the $60^{\circ}$ dislocations could separate to partials forming stacking faults of some kinds beetween them. And there may be other kinds of dis locations which could make a row along the small angle boundary. But, these possibilities should be considered precisely when more data about dislocations in ice crystal have been obtained in future; at this time the authors are obliged to be content with such a semi-quantitative interpretation by a simple gliding mechanism of dislocations.

\section{c) Effect of the stress on the rate of movement}

The multiplier terms of $1-y+\frac{1}{2} y \log y$ in eq. $(3)$ or $\sqrt{1-y} /(1+y)$ in eq. (4) indicate that the activation energy should decrease with increasing applied stress. But, the results graphed in Fig. 8 do not show this tendency. This may suggest that the simple mechanism of slipping motion of dislocations on the basal plane could not be the only mechanism of the movement of the small angle boundary. As the critical shear stress $\sigma_{0}$ has not yet been determined for ice crystals, a quantitative discussion can not be made further.

Another difficulty in explaining the results of experiments is that the rate of movement is not inverse proportional to the thickness of the specimen, in spite of the fact that it is proportional to the weight. If the applied stress on a cantilever of ice is assumed to be calculated by a simple elastic formula, it has an expression as follows at the neutral plane,

$$
\tau=\frac{3 P}{2 h}
$$

where $P$ is the load at the end of the cantilever and $h$ is the thickness of the bar. Therefore, if the rate of movement should be proportional to the stress, its dependency on the load and thickness must be proportional and inverse proportional respectively. Proportionality of the load $P$ holds quite well as shown in Fig. 9. But dependency on the thickness is largely different from the expectation as was shown in equations (1) and (2). It is not yet obvious from where this discrepancy comes. 


\section{d) Nature of the blocking barrier of dislocations}

Results of the experiments on the reversal of direction of movement seem to show that the sources of blocking dislocations for the movement on the basal plane increase with repetitions of the reversal, because the rate of movement under the same stress decreases with the ordinal number of the reversal. This is just opposite to the observations in the case of zinc, where the critical stress to move the boundary at constant rate decreases with the number of the reversal. ${ }^{12}$ Therefore, the nature of the blocking barrier of dislocations must be more or less different from those generally accepted, such as lattice vacancies or mixed dislocations of different orientations.

\section{e) Future problems}

As stated above, there are many peculiar observations of the movement of small angle boundaries of ice which could not be interpreted as examples of a dislocation mechanism accepted hitherto to explain the boundary motion in other crystals like zinc. For clarifying these points, it is necessary to know the activation energy for the creep of a single crystal of ice as well as the one for self diffusion in ice crystal. To form the basis of quantitative discussions of the direction dependency of the activation energy, the value of critical shear stress is needed. To meet these requirements, experiments on creep, self-diffusion and stress-strain relation are under way. Electron-microscope observations of dislocations in ice crystal are also progressing in order to ascertain the dislocation motion.

\section{Acknowledgements}

This investigation was financially supported partly by the Science Research Fund of the Ministry of Education. The authors wish to express their sincere appreciation to Dr. H. SuzUKI of the Japan Atomic Energy Research Instiugte for his valuable discussions and for allowing them to use his unpublished formulae. Their thanks are also due to Mr. T. Torzumi and Mr. K. InAfuku for helping with the experiments.

\section{References}

1) ReAD, W. T. JR. : Dislocations in Crystals. McGraw-Hill Book Co., Inc. New York, 1953.

2) Higashr, A. et al.: Glaciological Investigations of the Mendenhall Glacier, Alaska in 1960. The Arctic, to be published in 1962.

3) Butrovitch, T. R.: Density of Single Crystals of Ice from A Temperature Glacier. 
SIPRE Research Paper 7, 1953.

4) NAKAYA, U.: Properties of Single Crystals of Ice, Revealed by Internal Melting. SIPRE Research Paper 13, 1956.

5) HiGuchi, K.: The Etching of Ice Crystals. Acta Metallurgica, 6, pp. 636-642, 1958.

6) ITAGAKI, K.: Unpublished.

7) Muguruma, J. and A. HIgAshi : Electron-microscope Study of the Surface of Plastically Deformed Ice Crystals. To be published.

8) Humbel, F., F. JonA und P. ScFerRer: Anisotropie der Dielectrizitätskonstante des Eises. Helv. Phys. Acta, 26, pp. 17-32, 1953.

9) Butkovich, T. R. and J. K. LANDAUER: Creep of Ice at Low Stresses. SIPRE Research Report, 72, 1960.

10) SuzUKI, H.: Private Communication.

11) Glen, J. W. and M. F. Perutz: The Growth and Deformation of Ice Crystals. J. Glaciology, 2, pp. 397-403, 1954.

12) Li, C. H., E. H. Edwards, J. WAshburn and E. R. PARker: Stress-Induced Movement of Crystal Boundaries. Acta Metallurgica, 1, pp. 223-229, 1953. 